\section{RELATION OF CRYSTALLOGRAPHY TO CHEMISTRY}

\author{
By Dr. A. F. WELLS \\ Research Department, Imperial Chemical Industries \\ Limited, Manchester
}

$I^{N}$ the development of chemistry two phases can be distinguished. First there is a subdivision into more or less self-contained sections, inorganic, organic and physical-a subdivision dictated by the increasing complexity of the subject. Corresponding divisions have taken place in the other natural sciences, and the training of scientific workers has become more and more specialized. In the second phase the gulfs between the different sciences and between the branches of a particular science tend to be bridged by theories and new techniques. For example, the foundations of modern chemistry were laid when physicists investigated the passage of electricity through gases and the emission of radiation from heated bodies, studies which eventually led to the modern theory of the structure of the atom. Just as the quantum theory contributed to the development of chemistry as well as physics, so within chemistry itself the techniques of the physicist and the physical chemist are required by the inorganic and organic chemist. Thus although the various branches of chemistry have separately become more complex, they have now much in common as regards theory and technique.

In relation to chemistry the science of crystallography occupies a special position. Classical crystallography was restricted to the study of the external forms of crystals and their physical properties, though the study of crystals naturally led to speculations concerning the fine structure of matter, with the result that much of the space-group theory required in X-ray crystallography was available long before it was possible to use it. With the discovery (by physicists) of the diffraction of X-rays by crystals the whole status of crystallography was altered. From being essentially a descriptive subject, of comparatively little importance to chemistry, it gave rise to a new technique making possible the determination of the spatial arrangement of atoms. Moreover, the new structural crystallography is not limited, as was classical crystallography, to the study of well-developed crystals, for its methods may be applied to powders, to quasi-crystalline materials in which there is only partial ordering of the atorns, and even to glasses and liquids. It has become customary to describe that part of the new knowledge which is of more chemical interest as 'crystal chemistry', while 'crystal physics' embraces the study of the physical properties of crystals and their relation to internal structure. In this article I shall endeavour to show that the subject-matter of crystal chemistry is of such fundamental importance to chemistry that it must be incorporated into chemistry as an integral part of that subject.

The question of the relation of crystallography to chemistry is not only of academic interest. It is intimately concerned with the practical problem of how chemistry should be taught, and the type of training determines the mental outlook of chemists and hence the way in which the subject develops. Certain practical advantages of a knowledge of crystallography are sufficiently evident; for example, the ability to recognize crystalline forms in microchemical analysis and to identify solid compounds by means of their refractive indexes. The recent publication of a book on the optical properties of organic crystals is a sign of the increasing attention being paid to the use of crystallographic data for such purposes. We still await a systematic compilation of morphological data such as was envisaged many years ago by T. V. Barker. Indexes of optical, morphological and $\mathrm{X}$-ray data represent three ways in which crystallography can be of immediate practical value to chemists. The ability to 'think in three dimensions' and the appreciation of the relative shapes and sizes of groups of atoms are invaluable to those designing molecules of particular shapes required, for example, in modern synthetic polymer research.

We might make two generalizations about chemistry which illustrate the artificiality of separating crystal chemistry from chemistry. The first is that the chemist is chiefly interested in reactions between atoms, ions or molecules of different kinds; and the second is that so far as structural studies are concerned he deals only with finite groups of atoms. These limitations arise from the very nature of chemical methods and illustrate two essential differences between chemical and physical methods of studying a substance. The chemist studies it by changing it into something else; that is, he investigates how it reacts with other substances, the nature of the products, the mechanism of the reaction, and so on. Furthermore, he can deal only with substances in the liquid, dissolved or gaseous states, in which they exist as finite molecules or complex ions. When, therefore, an indefinitely large grouping of atoms has been formed, it can be studied only by breaking it down into smaller fragments which can then be examined by these chemical methods. The physicist, on the other hand, studies the way in which matter interacts with energy in some form or other without, in general, causing any irreversible change in composition.

In certain cases the chemist is interested in the combination of atoms of the same element, for example, when they form a finite molecule $\left(\mathrm{Cl}_{2}, \mathrm{P}_{4}\right.$, etc.), and the determinations of the numbers of atoms in these molecules were essential to the assignment of correct atomic and molecular weights. The cohesion of such molecules in the solid and liquid states involves only interactions of a feebler sort between the electronic systems of molecules within which the primary chemical valencies of the elements are already satisfied; that is, the crystal is built up of discrete molecules held together by van der Waals bonds. There are, however, other possibilities. For example, an element may form finite molecules in solution or in the vapour state, but further combination, by means of the same kind of bonds, takes place on crystallization, giving infinite arrays of atoms as in crystalline selenium (infinite chains) or black phosphorus (infinite layers). Some elements, indeed, have two erystalline modifications, one of which is built of finite molecules and the other of infinite 'molecules', as in the case of white and black phosphorus. In yet other cases there is no stable finite molecule, as with carbon and silicon. Not only similar atoms, but also similar molecules may join together to form more complex molecules. Where the latter are finite, the reaction comes within the province of chemistry; where infinite, the polymer is a solid. Thus molecules such as $\mathrm{Fe}_{2} \mathrm{Cl}_{6}$ and $\mathrm{Al}_{2} \mathrm{Cl}_{6}$ simply represent degrees of molecular complexity intermediate between the molecules $A X_{3}$ 
and the infinite 'molecules' in the solids. The formation of these intermediate molecules is the exception rather than the rule. As an example of another type of combination between similar molecules we may instance the dimeric forms of certain carboxylic acids. Here the two molecules are held together by hydrogen bonds of exactly the same type as hold together the molecules in the crystal. Just as there are two ways of building up an infinite array of molecules of oxalic acid by means of hydrogen bonds (into infinite chains and layers in the two crystalline forms), so in other cases there may be two ways of uniting the molecules, to give on one hand a finite dimeric molecule and on the other the infinite airray in the crystal. These examples should suffice to show that the comprehensive study of the interaction between atoms and molecules includes all cases of combination, whether the primary units be atoms or molecules of the same or of different kinds, and whether the product is a finite or an infinite grouping. We must now indicate other ways in which the study of the solid state has enlarged the scope of chemistry and contributed to our understanding of matters essentially chemical.

By enabling us to study atomic arrangement in the infinite arrays of atoms in crystals, X-ray crystallography has greatly extended the range of stereochemistry, a subject which hitherto had been restricted to the study of finite molecules and complex ions. We have already remarked that in order to study a substance by chemical methods it is necessary to use it in solution or in the vapour state, that is, to break down the extended groupings of atoms characteristic of the crystal into finite molecules or ions. Thus the information obtained from the study of the solid state is complementary to that provided by chemical methods, though the stereochemistry of finite molecules may also be studied-and with greater precision -in the crystal, for in molecular crystals the internal structure of the molecule is in general not appreciably affected by the relatively weak intermolecular forces. We may remark here that the physical methods have an important advantage, apart from their greater precision, over the chemical methods, in that they do not depend on deductions concerning the mechanisms of reactions, a complication inherent in the classical methods of studying stereochemistry. The study of crystals has had much less effect on organic than on inorganic chemistry, for a number of reasons. First, the structural units in crystals of the simpler organic compounds are generally the same finite molecules which are studied by the chemist. Second, most organic compounds are built from atoms of a small number of elements (carbon, nitrogen, oxygen, sulphur, hydrogen and the halogens), so that when the approximate disposition of bonds from these elements was known the rough configuration of quite complex molecules could be deduced. The third point is that relatively few complete crystal structure determinations of organic compounds have been made.

Inorganic chemistry, however, presents a very different picture. Not only have we to deal with a very large number of elements, but also there is the additional complication that many of them do not form molecules or complex ions amenable to study by the methods of classical stereochemistry, which required the preparation of molecules or complex ions exhibiting stereoisomerism or optical activity. Whereas, for example, the arrangement of bonds around a silicon atom had to be deduced from the optical activity of a compound such as $\left(\mathrm{C}_{2} \mathrm{H}_{5}\right)\left(\mathrm{C}_{3} \mathrm{H}_{7}\right)$ Si $\left(\mathrm{CH}_{2} \cdot \mathrm{C}_{6} \mathrm{H}_{5}\right) \quad\left(\mathrm{CH}_{2} \cdot \mathrm{C}_{6} \mathrm{H}_{4} \mathrm{SO}_{3} \mathrm{H}\right)$, and whereas the evidence for a particular arrangement of bonds might be only the feeble optical activity of a single compound, the stereochemistry of an element is often established with certainty by the analysis of the crystal structure of a very simple compound or of the element itself. Thus the tetrahedral arrangement of the four bonds from a silicon atom is evident from the structure of the crystalline element, and the planar disposition of the four bonds formed by divalent palladium or platinum is proved by the crystal structures of the chloride $\left(\mathrm{PdCl}_{2}\right)$ and sulphide (PtS) respectively. Since detailed crystal structure determinations have been made of many more inorganic than organic compounds, the whole aspect of the structural side of inorganic chemistry has been changed.

The effect of studying the spatial arrangement of atoms in crystals has been much greater, however, than simply to extend the subject-matter of stereochemistry and to make its data more precise. The detailed study of bond-lengths and inter-bond angles, together with the similar information gained by the application of other physical methods such as spectroscopy and electron diffraction, has added greatly to our knowledge of the nature of the bonds between atoms. In the realm of inorganic chemistry the study of the solid state has given us knowledge of the constitutions of many large groups of compounds which could not be studied by purely chemical methods. Prior to the development of X-ray crystallography, it was not possible to elucidate the structures of such groups of compounds as hydrates, silicates, etc., which exist only in the solid state, and the postulation of whole series of fictitious silicic acids is perhaps the best-known example of the result of making deductions about the constitutions of solids without having any knowledge of the atomic arrangement therein. The knowledge we have acquired of the principles underlying the structures of compounds of many types enables us to appreciate the limitations of empirical formulæ as means of expressing the constitutions of solids. For example, the compounds calcium carbonate $\left(\mathrm{CaCO}_{3}\right)$, silicate $\left(\mathrm{CaSiO}_{3}\right)$ and titanate $\left(\mathrm{CaTiO}_{3}\right)$ have quite different structures, the first containing discrete $\mathrm{CO}_{3}{ }^{2-}$ ions, the second cyclic $\mathrm{Si}_{3} \mathrm{O}_{9}{ }^{6-}$ ions, while the third contains no complex ions. The recognition that not only the satisfying of chemical valencies or the balancing of charges, but also geometrical factors play a part in determining the composition of a solid leads to a fuller understanding of concepts so fundamental to chemistry as the laws of chemical combination. If a substance, whether in the solid, liquid, or gaseous states, consists of stable finite molecules, then we expect to find the law of fixed proportions rigidly obeyed. Also, in the case of an ionic crystal such as solium chloride $(\mathrm{NaCl})$, the requirement of electrical neutrality necessitates the presence of equal numbers of sodium and chlorine ions. Although non-stoichiometric crystals can be prepared by special methods (for example, by heating a crystal of sodium chloride in sodium vapour) the departures from stoichiometric composition are very small. In the case of ferrous sulphide $(\mathrm{FeS})$, however, the sulphur lattice is sufficiently rigid to tolerate the absence of some of the iron atoms, with the result that the ' $\mathrm{FeS}$ ' phase is stable over the range $50-55.5$ atomic per cent sulphur, accounting for formulæ ranging from $\mathrm{Fe}_{6} \mathrm{~S}_{7}$ to $\mathrm{Fe}_{11} \mathrm{~S}_{12}$ assigned to natural specimens of this 
material. In this connexion it is interesting that ferrous sulphide is so often quoted in elementary text-books as an example of a chemical compound, and that it figured in the controversy between Proust and Berthollet over the law of fixed proportions. In 1804 Berthollet expressed the view that "the metals can combine with sulphur in very various proportions, and the combinations which are thus formed have very different properties according to their proportions : I am, in this case, again in opposition to the opinion of Proust, who asserts that by the invariable law of proportions sulphur and iron are fixed at $60: 100$ ". It would seem fortunate that these early investigators studied the system Fo-S rather than $\mathrm{Ni}-\mathrm{Te}$, for example, in which phases with all compositions ranging from $\mathrm{NiTe}$ to $\mathrm{NiT}_{2}$ are stable. Here, however, we are nearer alloys than chemical compounds; but this only serves to illustrate another point. No definite dividing line can be drawn between alloys and chemical compounds, and alloys-particularly the 'interstitial compounds' (certain hydrides, borides, carbides and nitrides)are notable for their variable compositions.

So far we have been considering to what extent a knowledge of the arrangement of the atoms in the interior of crystals affects our outlook on chemistry. This knowledge is, however, also essential to an understanding of the properties of the surfaces of the crystal and of processes involving the formation or breakdown of the crystal. Reactions involving a solid phase must start at the surface of the solid, and such reactions are of particular interest both theoretically and practically. The oxidation of a solid metal was one of the first reactions studied quantitatively (the increase in weight of metallic tin on oxidation was recorded by Jean Rey in 1630); but the investigation of the mechanisms of such processes is difficult, and it is only recently that much progress has been made in this field. For example, the results of studying the dependence on oxygen pressure of the semiconductivity of oxides such as cuprous oxide and zinc oxide, and of the rates of oxidation of the corresponding metals, suggest that these phenomena are closely related. In the case of the oxidation of ferrous oxide $(\mathrm{FeO})$ through $\mathrm{Fe}_{3} \mathrm{O}_{4}$ to $\mathrm{Fe}_{2} \mathrm{O}_{3}$, we can now give some sort of physical picture of the process on the atomic scale. The activity of many surfaces is well known and is made use of in a variety of ways. The properties of a surface must be accounted for, not only in terms of the properties of the individual atoms or molecules composing the surface, but also of the atomic structure of the surface, that is, the actual arrangement of atoms in the surface and the difference between the environment of a surface atom and a similar one in the interior of the crystal. The phenomena of crystal growth-the development of different habits from different solvents, the effects of impurities on the habits of crystals and more generally of the form in which a solid separates from solution, whether crystalline or colloidal-all these are associated with the properties of atoms in surfaces and with the way in which the solute interacts with the solvent. The solubility of one substance in another is evidence of interaction between the two substances, and this may be of various kinds. For example, the interaction between molecules of naphthalene and benzene or between stannic iodide and earbon disulphide is quite different from that between, say, resorcinol and water.

The term 'soluble' is loosely used in various senses in chemistry; for example, when it is said that sodium chloride is soluble in water, ferric chloride in pyridine, mercuric iodide in a solution of potassium iodide or finally a metal in an acid. Even when no obvious chemical reaction takes place, there may be important alterations in the constitution of the solute. Thus in solid ferric chloride there are infinite groupings of iron and chlorine atoms in which every iron atom is surrounded by six chlorine atoms. If this compound is dissolved in carbon disulphide, it forms molecules of $\mathrm{Fe}_{2} \mathrm{Cl}_{6}$, as it does in the vapour at temperatures below $750^{\circ} \mathrm{C}$., but in solution in pyridine the condensation $2 \mathrm{FeCl}_{3} \rightarrow \mathrm{Fe}_{2} \mathrm{Cl}_{6}$ is prevented by the linking of a pyridine molecule to each iron atom. Thus in these various cases $w \theta$ find that 'ferric chloride' exists in quite different forms with iron atoms bonded to 6,4 and 3 chlorine atoms respectively.

There is much more to be known about a compound, therefore, than the mode of preparation and its chemical properties. It has a structural chemistry of its own if we include within the province of structural chemistry the relations between the constitution of a substance in different states of aggregation. Finally we may mention the interpretation of data concerning processes which involve the formation or breakdown of crystals. The analysis of data such as heats of fusion, sublimation and crystallization calls for a knowledge of the actual processes taking place when the crystal melts, vapourizes or is built up, in terms of the nature and numbers of bonds broken or formed.

In conclusion, we would suggest that there is no longer any justification for drawing a dividing line between finite and infinite arrangements of atoms, and regarding the study of the former as constituting chemistry and of the latter crystal chemistry. The chemist has concerned himself exclusively with finite groups of atoms because he must break down the infinite groups of atoms in crystals before he can apply his chemical methods to them. The tendency to regard all compounds as existing in the form of finite molecules arose quite naturally from the study of gases, which necessarily exist in this form, and from the intensive study of organic chemistry, which is concerned with substances existing for the most part as finite molecules in all states of aggregation. If the study of atomic arrangement in the solid state had become possible at an earlier stage, the scope of chemistry would not have been limited in the ways we have been discussing. We have seen that as regards inorganic compounds, although it is true that some crystals may be broken down and reformed reversibly (for example, simple salts), while with others the breakdown of the crystal destroys the chemical identity of the compound (as in the case of silicates), yet in all cases there is a wealth of information which can be gained only by studying the infinite arrays of atoms which constitute the solid compounds. Similarly, the fact that the molecules which exist in a crystal of glycine may be studied in solution is no reason for neglecting to study the way in which they are held together in the solid state. In the attack on the structures of substances of increasing complexity, from the large finite molecules such as globular proteins and viruses to the infinite 'molecules' of fibres, natural and synthetic, the chemist must rely more and more on the crystallographer. When building up a chain step by step (for example, $2 \mathrm{CH}_{3} \mathrm{I}+$ $2 \mathrm{Na} \rightarrow \mathrm{C}_{2} \mathrm{H}_{8}+2 \mathrm{NaI}$ ) the chemist could deduce the constitution of the product. When, however, he polymerizes simple aliphatic compounds to polymers of high molecular weight, for example, ethylene to 
polythene, he can no longer determine the structures of his solid products and must rely on the X-ray crystallographer to tell him what he has made. The old chemical methods of synthesis and degradation give only limited information about the structural units, and to discover how they were originally linked together is not always within their power.

\section{COMMENTS ON CHROMOSOME STRUCTURE}

\section{By Dr. I. MANTON \\ University of Manchester}

$\mathrm{W}$ HEN a great physicist takes the trouble to explain in simple language some of his matured thoughts on topics of general interest outside his own subject, it is an event for which one eannot be too grateful. The following remarks have been aroused by the appreciative perusal of Prof. E. Schroedinger's delightful little book "What is Life ?"1.

Without attempting to summarize the whole of Prof. Schroedinger's argument, it is valuable to notice the great stress which is laid on the existence of two very different methods of obtaining orderly behaviour of matter in Nature. In the inanimate world 'order from disorder' is said to be the rule; the behaviour of matter in bulk being in most cases the expression of a statistical average of the behaviour of vast numbers of particles (atoms, molecules or the like) which, individually, may be doing the most diverse things under the sole compulsion of a tendency towards increased randomness. In biological systems, on the other hand, 'order from order' is met with. In such a system the most complex sequence of events may be determined and set in motion by the pattern of arrangement in space of a comparatively minute number of individual particles occupying relatively fixed positions with regard to one another. The paramount importance of the pattern of atomic arrangement in the particular case of the genetical material carried by the chromosomes is, in Prof. Schroedinger's view, the most interesting discovery of our time.

Few biologists will probably wish to dispute this in general terms. Cytological comment is, however, aroused by the details of its presentation from the circumstance that Prof. Schroedinger, at various points, is thinking in terms of certain assumptions regarding chromosome structure which are by no means universally held. It may therefore be of interest to inquire what change of view, if any, will be entailed if these assumptions are altered.

Prof. Schroedinger is much impressed by the singleness of the 'code script' in inheritance, by code script meaning the sum of hereditary material carried by a haploid nucleus (in genetical parlance this would be referred to as a genome). That only one chromosome set or genome is actually necessary for development is clearly shown by the existence of haploid organisms, for example, many of the lower plants, or cases of parthenogenesis in both plants and animals; it is therefore quite legitimate to ignore diploidy and polyploidy. Difficulties, however, appear at once if 'singleness' is interpreted literally in a molecular sense, and that this is Prof. Schroedinger's interpretation seems clear from his very interesting discussion of the size of a gene.
It might perhaps be questioned whether the "size of a gene' is a desirable or legitimate use of words. In its original sense a 'gene' meant nothing more than the physical basis of an externally visible mutation, and if a mutation can be caused, as Prof. Schroedinger suggests, by a change of atomic arrangement, of the nature of a quantum jump, occurring within a molecule, then a gene, strictly speaking, is the changed part of that molecule and nothing else. It is an unfortunate biological practice which Prof. Schroedinger cannot be personally blamed for following, that the word is now often used in so many extended senses that it has little precise meaning left. In discussing the maximum size of a 'gene' from genetical data, the word denotes either the smallest piece of a chromosome which can have a genetically detectable effect, or the shortest distance between two mutations which can be separated by crossing-over; a numerical estimate of either of these by existing genetical or cytological methods may be expected to yield purely subjective values expressing present crudities of technique. In discussing the minimum size of a 'gene' as deduced from induction of mutations by ionizing radiations, the word is apparently equated with the range of influence of the minimum degree of ionization required to induce a mutation. The thing that Prof. Schroedinger really wishes to discuss is the fundamental molecular unit of chromosome structure, which is not necessarily identical with any of these concepts. The use of the word gene for this also may seem particularly unfortunate to a cytologist because some idea of structural discontinuity is almost inevitably implied. Mutations are discontinuous and arranged in linear sequence along a chromosome. Whether the fundamental genetical material in which the mutations occur is or is not also discontinuous (like beads on a string) is quite another question and one which neither cytology nor genetics can yet determine. This linguistic difficulty would perhaps best be met if the word gene were deleted from the vocabulary; one of the most pregnant of Prof. Schroedinger's sentences could then be paraphrased as- "We believe a mutation to be of the nature of a quantum jump and the fundamental unit of chromosome structure-or perhaps the whole chromosome fibre-to be an aperiodic solid".

At this point the sense in which the "whole chromosome fibre" can be regarded as a unit becomes of importance. There are at present two extreme views as to this, both supported by some positive evidence. According to the one which Prof. Schroedinger is using, the whole chromosome thread is a single structure at all times, except for a limited period during prophase when it is doubled in preparation for a nuclear division. The strongest piece of evidence in support of this is the differential behaviour of certain nuclear stages to irradiation by $\mathrm{X}$-rays at dosages sufficient to cause gross chromosome fracture. Assuming that the statements in the literature are correct (for example, Riley $1936^{2}$ ) the position appears to be that in some organisms such as Tradescantia ${ }^{3}$, irradiation at prophase can cause fracture of halfchromosomes (chromatid breaks) but at other stages only breakage of whole chromosomes. In interpreting this as meaning that the chromosome thread is single at all stages other than prophase it is not always realized that this singleness could be conferred by spiral strueture and is not necessarily based on singleness of the genetical material.

The spiral structure of chromosomes (or perhaps more correctly the helical structure, since the geo- 